\title{
Wearable Wireless Sensing for Sports and Ubiquitous Interactivity
}

\author{
Michael Lapinski, Mark Feldmeier, and Joseph A. Paradiso \\ Responsive Environments Group \\ MIT Media Lab \\ Cambridge, MA 02139 -USA- \\ mtl@media.mit.edu, carboxyl@mit.edu, joep@media.mit.edu
}

\begin{abstract}
In this paper, we overview recent work from our research group that explores two very different applications of wearable inertial systems. The first project exploits an array of wearable, ultrawide-range, synchronous IMUs to measure the performance of professional baseball players. We describe some special aspects of our hardware (a dual-range, 6-DOF IMU with magnetometer), and show sample data from our current analysis. We also overview another project where we leveraged wearable sensors, including a micropower integrating accelerometer, for mobile personalized comfort control of building HVAC (heating/air conditioning) systems.
\end{abstract}

\section{INTRODUCTION}

Inertial sensors have followed corollaries of Moore's Law, benefitting from consistent advances in microfabrication technologies and denser electronics integration. Accordingly, multiaxis inertial sensors have become steadily cheaper (e.g., 3-axis accelerometers currently price in commodity under $70 \notin$, which will drop by more than a factor of two by 2015 [1]), and tend to require less power (e.g., under a milliamp for common triaxial accelerometers, soon to be single-digit microampres). Accordingly, inertial sensors, once the exclusive province of high-end applications like aerospace, now become commodity components (accelerometers \& magnetometers are projected to be integrated into half and gyros into $20 \%$ of all cellphones within 5 years [1]), and the applications that now drive them are mainly consumer oriented, such as user interfaces, gaming control, phone/tablet augmented reality, and health/fitness.

The Responsive Environments Group at the MIT Media Lab has been using inertial devices in wearable sensing and Human Computer Interfaces (HCI) applications for the last 15 years [2]. Starting in 1996 with a multimodal handheld conducting baton [3] bearing some similarity to the subsequently well-known Wii game controller, we have integrated inertial and other types of kinematic sensors into most of our projects. This article overviews two very diverse examples of our more recent forays into inertial applications sports medicine and wearable management of air conditioning (HVAC) systems for dynamic comfort control.

\section{INERTIAL SENSING FOR BASEBALL}

A primary tool of biomechanical analysis in sports medicine has been the optical tracker. Sports laboratories across the world are equipped with commercial vision-based marker trackers similar to those used ubiquitously in the motion picture industry. Although these devices can track position fairly accurately, they exhibit significant drawbacks that limit their utility for sports analysis. For example, they tend to require considerable time to set up and calibrate, take up a large dedicated area, can not be trivially moved, and are sensitive to background IR light and reflections. Additionally, they tend exhibit limited sampling rates (e.g., $200 \mathrm{~Hz}$ ) when run in their default mode, and can yield significant error when calculating forces and torques, which involve a doubledifferentiation of position measurements that can be quite sparse when an athlete is performing an extreme motion. Wearable inertial sensors, on the other hand, can be quickly put onto a player anywhere, are unaffected by background light, exhibit bandwidth well exceeding $1 \mathrm{KHz}$, and proportionally infer forces and torques without noisy differentiations. Accordingly, we have developed a suite of wearable inertial sensors for applications in sports medicine.

This project had its genesis in a wearable system of wireless IMUs called "Sensemble" [4] that we originally developed for interactive dance ensembles, but subsequently modified to exhibit the extended sensor range needed for sports measurements. This launched an ongoing collaboration with the Sports Medicine Department of the Massachusetts General Hospital to develop and apply a series of wearable wireless inertial sensors for sports medicine that we currently call the "Sportsemble" system [5].

Figure 1 shows our current Sportsemble node, each of which has three single-axis $\pm 120 \mathrm{G}$ ADXL193 accelerometers, three single-axis ADRX300 gyroscopes, three axes of low-rate gyros (using Invensense devices), a 3-axis $\pm 8 \mathrm{G}$ LIS344ALH accelerometer, and a HMC6343 digital magnetometer. The gyros, which normally saturate at $\pm 300 \%$ s were specially biased and strapped to respond up to $12,000 \%$ s, as outlined in [5]. The multi-range accelerometers and gyros let us record 
slow motion with the low-G devices and fast motion with the high-G units, thus providing high relative resolution across an entire athletic gesture (an IMU with logarithmic sensitivity would be appropriate for this application). The current Sportsemble uses an AVR32 processor with removable SD memory cards - all data is stored locally, then transferred to mass storage at the end of each day. The embedded radio (a $2.4 \mathrm{GHz}$ Nordic nRF2401a, which has a maximum bandwidth of $1 \mathrm{Mbps}$ and an output power of $+4 \mathrm{dBm}$ ) is mounted on a daughter card to isolate the RF electronics from the main board and enable an easy upgrade of the RF hardware. The radio protocol is a custom-designed lightweight TDMA scheme [5], and is primarily used now to synchronize all nodes to better than $1 \mathrm{~ms}$, demark different test runs, and monitor diagnostic information that's indicative of node health. Sportsemble nodes use a $145 \mathrm{mAh}$ lithium polymer rechargeable battery that can continuously power a node for circa 3 hours of use. The nodes are continuously active when switched on - although adaptive power management techniques can reduce the average needed current, this degree of node longevity is adequate for our typical testing session. The Sportsemble node is currently being revised - our new device will be smaller, using onboard flash memory accessible via a USB connection, an upgraded processor, possibly a triaxial low-rate gyro, and probably an AT89RF231 radio.

Under guidance from our sports-medicine physician collaborators, we now mount 5 nodes on players (we use both pitchers and batters), as shown in Fig. 2. Nodes are placed on the wrist, the forearm, the upper arm, the chest, and the waist. When a batter performs free swings, we mount the waist node onto the end of the bat - we don't mount an IMU on the bat when the batter hits the ball to prevent node damage (the strike instant can be readily ascertained from the wrist data, however, as seen below and elucidated in [5]). We now use a set of Neoprene straps custom-designed for us by an orthotics manufacturer to fix the sensors to the players. The straps have snug pockets for the sensor nodes - once the sensors and straps are attached, we secure them additionally with several layers of conventional sports pre-wrap tape (Fig. 3). The entire process (mounting/taping nodes and noting their position with a tape measure) takes only a few minutes.

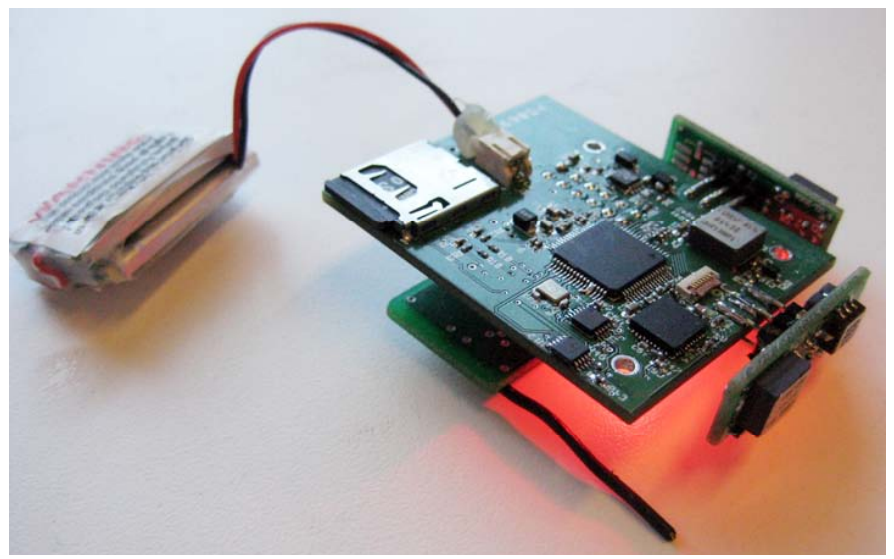

Figure 1. A current Sportsemble node, with rechargable battery. The two orthogonal daughter cards contain single-axis inertial components, needed for the extreme range, as multi-axis components generally don't extend to high G's and rates. The radio card is at bottom.
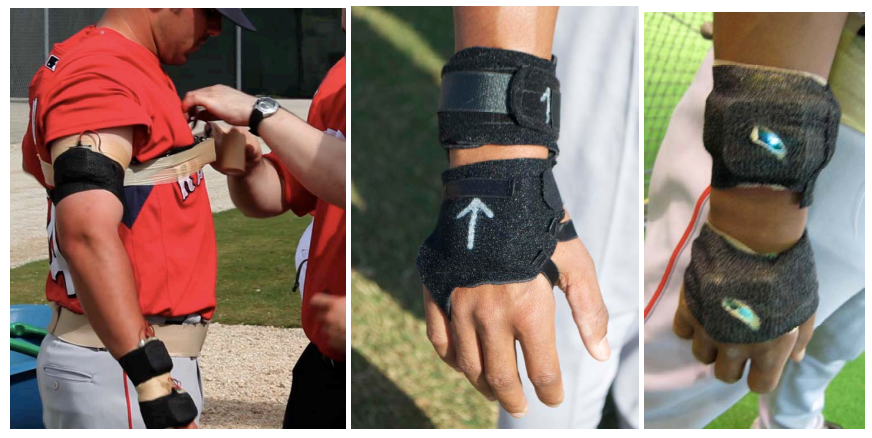

Figure 2. Attaching the Sportsemble nodes to baseball players. The nodes sit in custom Neoprene straps (center) that are taped over with Sports Wrap (left) to form a firm attachment (right).
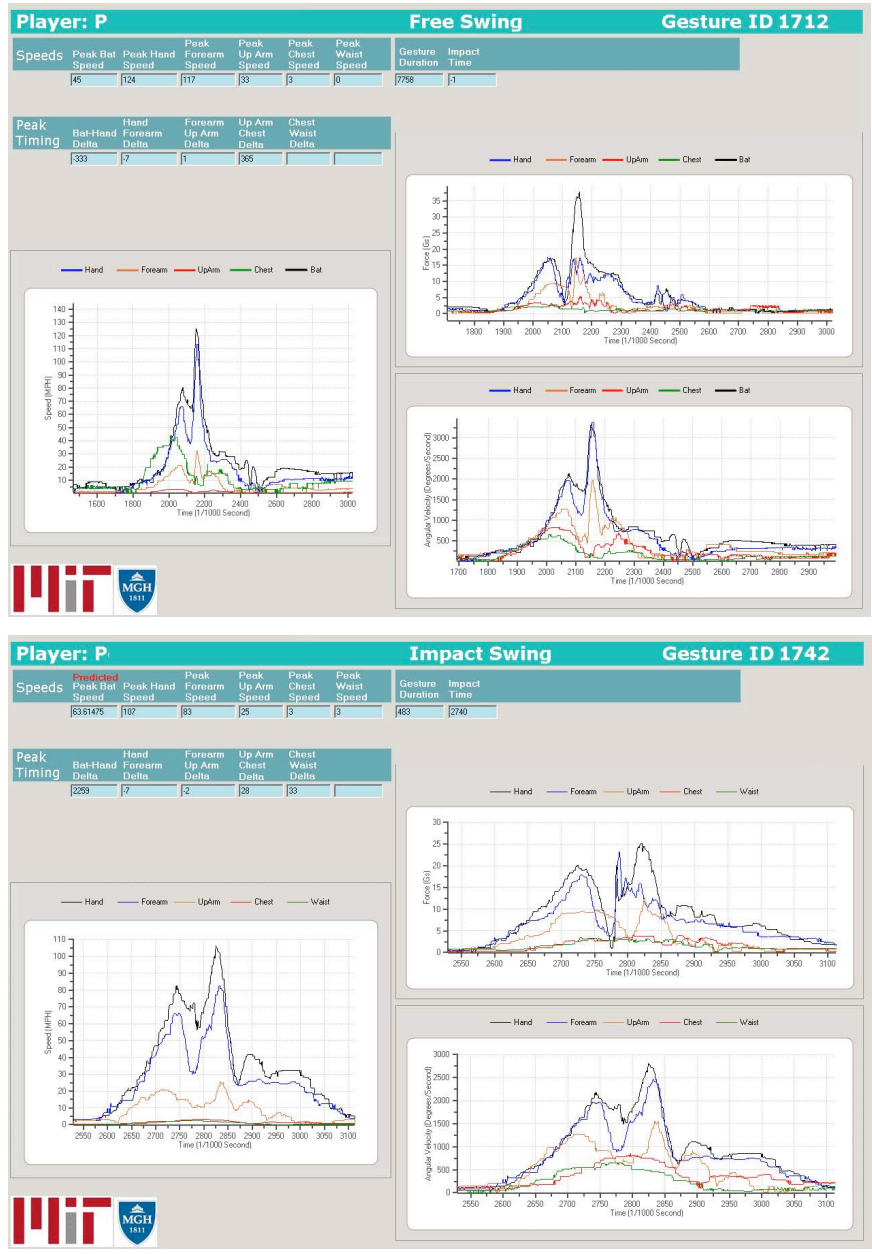

Figure 3. Batting examples from our database - inertial sensor data from a free swing (top) and a swing where the player hits the ball (below).

We have measured many professional players during the last few sessions of spring training (a total of 45 players and 1026 swings or pitches), and are collaborating with our sports medicine colleagues in extracting biomechanically-relevant parameters from this dataset. Our last hardware revisions rectified the lowpass filter problem mentioned in [5], and our data now has full bandwidth. Figures 3 and 4 show sample data from last year's spring training. Fig. 3 shows data from a 
batter. Speed is inferred by integrating the accelerometers, "force" is the measured accelerations, and angular velocity comes directly from the gyroscopes. The point where the player hits the ball can be clearly noted by the abrupt transient in the lower figure. Plots for a pitcher are shown in Figure 4. One can clearly note the wide range of measurement required by this application- players are seen to exhibit peaks of 8000 $\%$ s and 130 G's during a pitch.

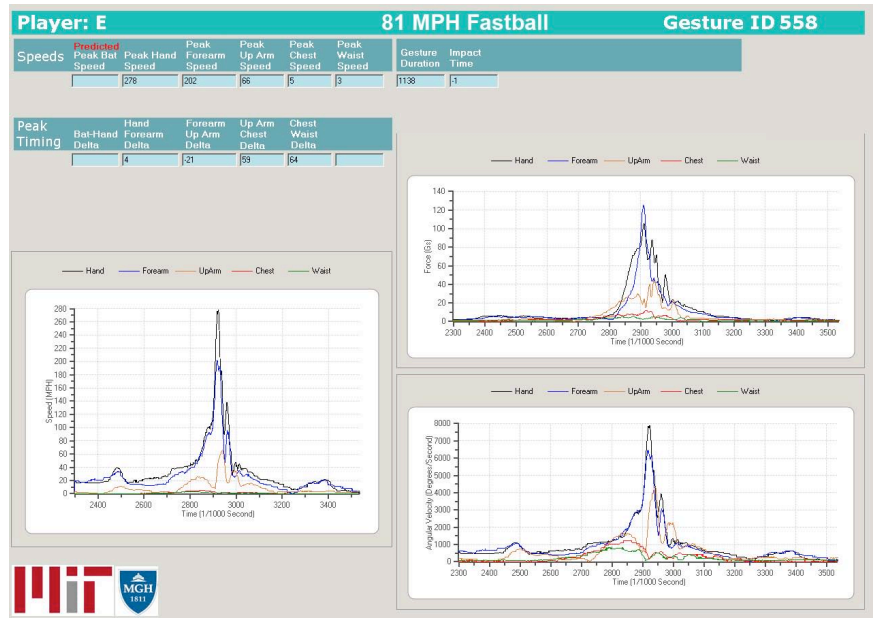

Figure 4. A pitching example from our database.

\section{HVAC COMFORT CONTROL}

Conventional heating and air conditioning systems regulate to temperature settings programmed into discrete thermostats, generally placed on a wall in the room that is to be heated or cooled. Although active thermal regulation in buildings gets gradually smarter, utilizing occupancy information and programmed setbacks, for example, air handling is still set around the notion of temperature measured at a set of fixed points, rather than occupant comfort.

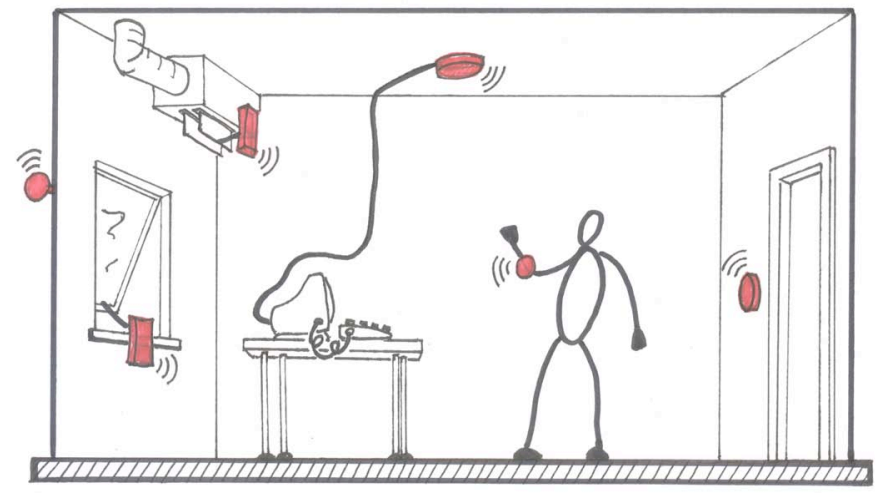

Figure 5. Elements of Distributed Comfort Control System.

A recent project in our group [7] has prototyped a system to explore this notion of dynamic comfort control. Figure 5 shows the elements of this system in one of the test rooms that we instrumented. We retrofit hardware to automatically open the windows or adjust the damper valve on the air conditioning vent in response to automatic control based on an ensemble of sensors in the room, outside the building, and worn on the user.

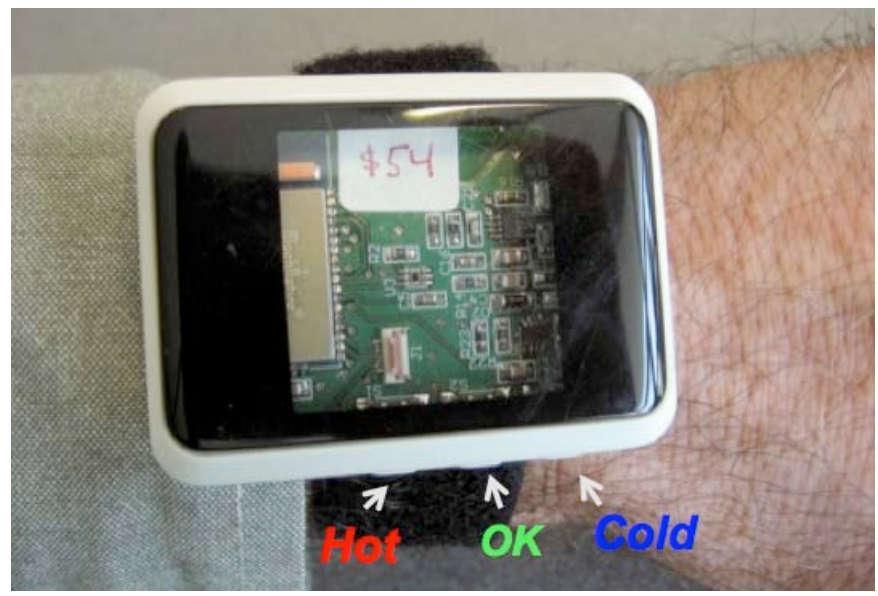

Figure 6. The wearable HVAC Comfort Sensor Node.

Figure 6 shows the wearable sensor unit, which contains an 802.15.4 transceiver/processor module, a light sensor, a temperature/humidity monitor, and an integrating micropower accelerometer. The device wakes once per minute to measure all sensor values and read/reset the activity integrator - if a compatible Zigbee network is detected, it uploads its data (in the absence of network coverage, the node caches up to 15 minutes of the most recent data, which would be sent when the network was recovered). The wearable node weighs $30 \mathrm{~g}$, and measures $54 \mathrm{~mm}$ by $40 \mathrm{~mm}$ by $14 \mathrm{~mm}$ when packaged (see Figure 6). It also has low average power consumption $(11 \mu \mathrm{A}$, giving a two year coincell battery life). This power drain is dominated by the clock used by the microprocessor module use of an appropriate external clock would reduce power draw by a factor of two. One of our early studies concluded that this node gave the most reliable data when mounted on the wrist [8], hence it was fashioned into a wristwatch form factor - better design could easily shrink it to the dimension of a small watch or build it into a watch.

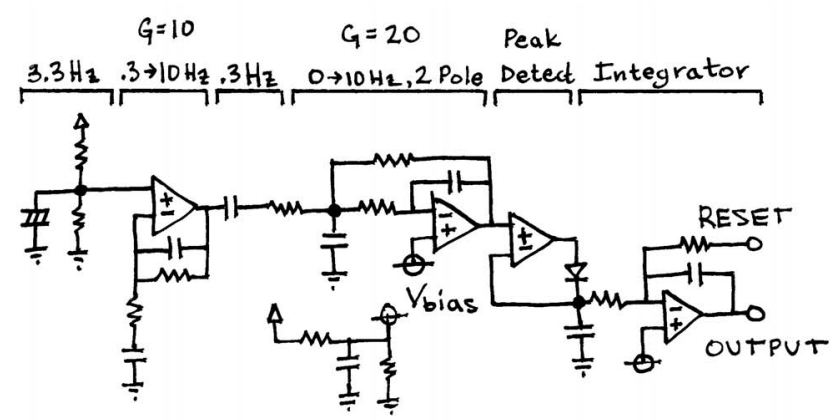

Figure 7. Micropower integrating analog accelerometer circuitry.

Although digital accelerometers begin to dramatically reduce their power requirements (e.g., [6]), active accelerometers were still far too power hungry for our application when this unit was designed, hence we developed our own micropower integrating accelerometer system based around a passive PZT cantilever chip, such as commonly used in the disk drive industry to detect drops or impacts. Figure 7 shows this design, consisting of a quad micropower operational amplifier conditioning the signal from a Murata Piezotite passive shock sensor. The active analog circuitry 
applies filtering, gain, rectification, and performs continuous integration (sensitive between 0.1 and $10 \mathrm{~Hz}$ ) for only $2-3 \mu \mathrm{A}$ current drain. The integrator is read and reset when the processor does its regular wakeup - if the integrator saturates beforehand, the processor can be interrupted.

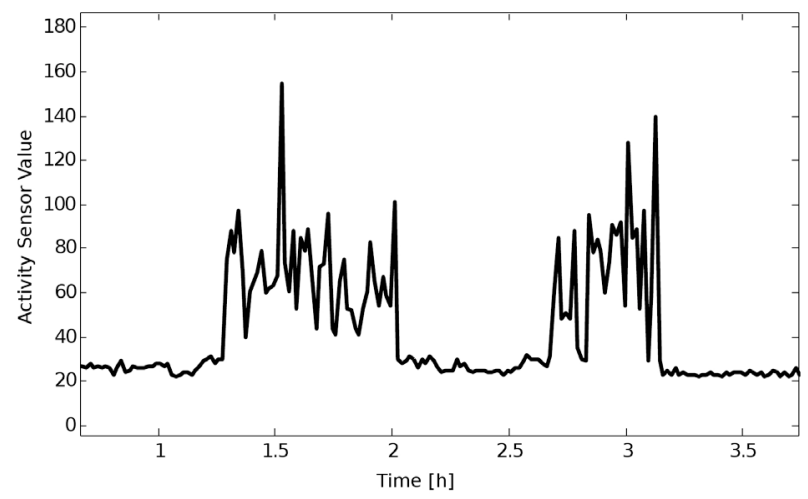

Figure 8. Integrated accelerometer data over 4 hours, showing clearly where the sensor was worn and removed.

The accelerometer is used to infer the level of activity of the user (Fig. 8) - e.g., in both a binary sense (whether the device is being worn or is left on the table) and in a continuous sense (how much is the user moving on average). The light sensor was included to infer ambient light levels or perhaps whether the user was wearing longsleeved clothing, and the temperature/humidity sensor was used to ascertain the user's thermal environment. Radio signal strength was found to be adequate to determine the room in which the user was located $[7,8]$, enabling their data to influence control of the local windows and HVAC vents.

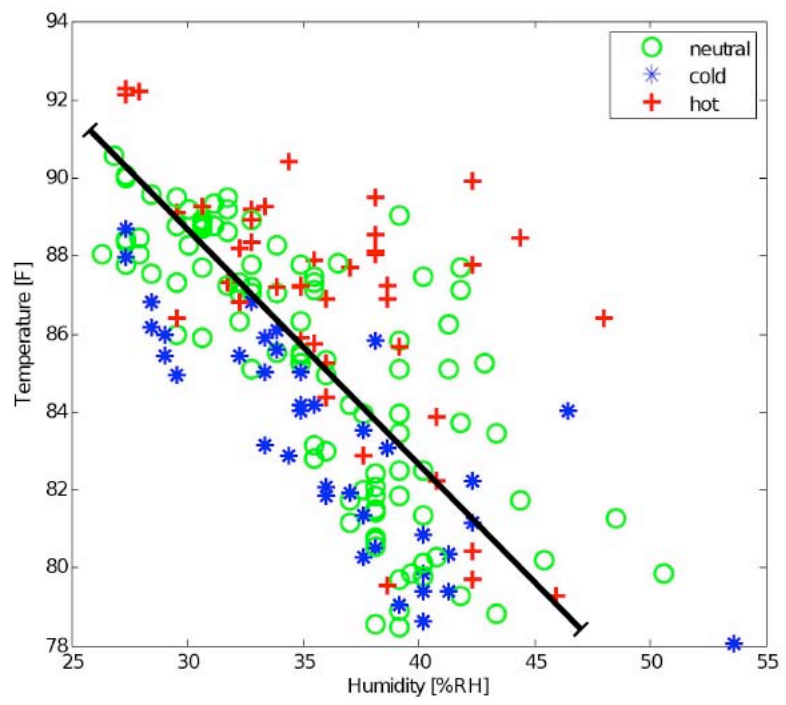

Figure 9. Temperature/humidity data for button pushes across a collection of users - blue was too cold, red too hot, and green neutral.

Each wrist unit had 3 buttons with which the user could declare their current level of comfort - a red button was for telling the system that you were too hot, pressing a blue one told the system that you were too cold, and the middle button was "neutral," indicating that you were comfortable. The button data was used to label the sensor data - when closed- loop control was activated, each user's level of comfort could then be ascertained from the sensor data $[7,8]$ and used to drive the HVAC. The main comfort discriminant came from the temperature/humidity data, as shown in Fig. $9-$ comfortable vs. uncomfortable points could be adequately separated for nearly all users via a simple linear classifier.

When the system was put into use during the last month of a Boston summer, much less chilled air was used (inferring a roughly $25 \%$ energy savings [7]), and users were much more comfortable than before on average (even though the air conditioning system was certified to be operating properly), indicating that systems of this sort have a promising future.

\section{CONCLUSION}

This paper summarized two very different recent projects in our research group that leverage wearable inertial systems in very different ways. Both projects are now evolving quickly - we are following baseball players to ascertain indicators that can point to impending injury, and we are designing a wristband controller that will integrate many of our interactivity projects, ranging from the smart HVAC work described here to other projects aimed at interaction with ubiquitous multiscale media [9] and smart lighting [10]. This wristband will allow us to test and evolve our ideas at a much greater scale, e.g., across an entire building.

\section{ACKNOWLEDGMENT}

We thank our other colleagues in the Media Lab's Responsive Environment Group who contributed to the work overviewed here, along with our collaborators in sports medicine at Mass General Hospital and in major-league baseball. This work was funded by the Media Lab's research consortia and a gift from the Boston Red Sox Foundation.

\section{REFERENCES}

[1] L. Robin, "Consumer electronics turn to MEMS for gesture control, precision location," EE Times, Online News \& Analysis, July 28, 2011.

[2] J.A. Paradiso, "Some Novel Applications for Wireless Inertial Sensors," in Proc. of NSTI Nanotech 2006, Vol. 3, Boston, MA, May 711, 2006, pp. 431-434.

[3] T. Marrin and J. Paradiso, "The Digital Baton: a Versatile Performance Instrument," Proc. ICMC 1997, Thessaloniki, Greece, September 1997, pp. 313-316.

[4] R. Aylward and J.A. Paradiso, "A Compact, High-Speed, Wearable Sensor Network for Biomotion Capture and Interactive Media," in the Proc. of IPSN 2007, Cambridge, MA, April 25-27, 2007, pp. 380-389.

[5] M. Lapinski, E. Berkson, T. Gill, M. Reinold, and J.A. Paradiso, "A Distributed Wearable, Wireless Sensor System for Evaluating Professional Baseball Pitchers and Batters," in Proc. of ISWC 2009, Linz, Austria, September 4-7, 2009, pp. 131-138

[6] For example, see the data sheet for the Analog Devices AD22365.

[7] M. Feldmeier and J.A. Paradiso, "Personalized HVAC Control System," in Proc. of IoT 2010, Tokyo Japan, Nov. 29 - Dec. 1, 2010.

[8] M. Feldmeier, Personalized building comfort control, Ph.D. dissertation, MIT Media Lab, 2009.

[9] M. Laibowitz, N-W Gong, and J.A. Paradiso, "Wearable Sensing for Dynamic Management of Dense Ubiquitous Media," in the Proc. of BSN 2009, Berkeley, CA, June 3-5, 2009, pp. 3-8.

[10] J.A. Paradiso, M. Aldrich, N. Zhao, "Energy-efficient control of solidstate lighting," SPIE Newsroom, March 252011. 